\title{
Revealing Multiple Geological Scenarios Through Unsupervised Clustering of Posterior Realizations from Reflection Seismic Inversion
}

Gulbrandsen, Mats Lundh; Cordua, Knud Skou; Hansen, Thomas Mejer; Mosegaard, Klaus

Published in:

Geostatistics Valencia 2016

Publication date:

2016

Document version

Peer reviewed version

Citation for published version (APA):

Gulbrandsen, M. L., Cordua, K. S., Hansen, T. M., \& Mosegaard, K. (2016). Revealing Multiple Geological Scenarios Through Unsupervised Clustering of Posterior Realizations from Reflection Seismic Inversion. In Geostatistics Valencia 2016 (Vol. 2, pp. 79-95). Universitat Politecnica de Valencia. 


\section{Revealing Multiple Geological Scenarios Through Unsupervised Clustering of Posterior Realizations from...}

Article · September 2016

\section{CITATIONS}

0

4 authors, including:

Mats Lundh Gulbrandsen

University of Copenhagen

9 PUBLICATIONS 1 CITATION

SEE PROFILE

Thomas Mejer Hansen

University of Copenhagen

64 PUBLICATIONS 407 CITATIONS

SEE PROFILE
READS

69

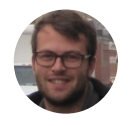

Knud Skou Cordua

Technical University of Denmark

41 PUBLICATIONS 199 CITATIONS

SEE PROFILE

Some of the authors of this publication are also working on these related projects: 


\title{
Revealing Multiple Geological Scenarios Through Unsupervised Clustering of Posterior Realizations from Reflection Seismic Inversion
}

\author{
Mats Lundh Gulbrandsen ${ }^{1}$, Knud Skou Cordua ${ }^{1}$, Thomas Mejer Hansen ${ }^{1}$, \\ Klaus Mosegaard ${ }^{1}$
}

\begin{abstract}
In this study, we analyze 26000 posterior realizations obtained through Monte Carlo sampling from the posterior distribution of a reflection seismic inverse problem and show that the posterior realizations cluster around multimodal peaks. This problem is based on a seismic trace recorded in the southern part of Jutland, Denmark. Prior information is based on observations of lithology sequences of the geology in the area, and the multimodal modes in the posterior realizations will hence represent different geological scenarios. In order to uncover the multimodal nature of the posterior distribution, grouping of posterior realizations is done using an unsupervised clustering technique, namely the K-means clustering algorithms. In order to quantify the choice on the number of clusters in the realizations, the gap-statistic-method is used. The clustering method is applied on both categorical model parameters representing lithological units, and on the continuous parameters representing the acoustic impedance. These techniques allow quantifying the probability of the different possible geological scenarios that are consistent with the seismic and geological observations. Results demonstrate that the cluster characteristics are significantly dependent on the types of parameters considered. If the goal of the inversion is to identify different geological scenarios using a parameterization based on lithological units is more informative than a parameterization based on acoustic impedance.
\end{abstract}

\section{Introduction}

A widely used approach for inversion of reflection seismic data is based on a linear forward model, and Gaussian assumptions about the prior distribution of the elastic parameters and the noise model, e.g. (Buland \& Omre, 2003), (Jullum \&

\footnotetext{
${ }^{1}$ Solid Earth Physics, University of Copenhagen, Juliane Maries Vej 30, 2100 Copenhagen

${ }^{2}$ The hamming distance is the number of position (coordinates/elements) that differs
} 
Kolbjørnsen, 2016), (Hampson, Russell, \& Bankhead, 2005). The solution to such inverse problems is Gaussian distributed, which is simply a single-modal distribution.

In reality, the Gaussian prior assumption and the linear forward model may not adequately describe the information available. In order to handle this, these problems can instead be formulated probabilistically, which leads to a solution described by a typically unknown and non-Gaussian posterior distribution, e.g. (Larsen, Ulvmoen, Omre, \& Buland, 2006), (Ulvemoen \& Omre, 2010), (Zunino, Mosegaard, Lange, Melnikova, \& Hansen, 2014), and (Bosch, Rodrigues, Navarro, \& Díaz, 2007). In this way, the solution to the inverse problem can be characterized by a sample from the posterior distribution, which will represent a set of realizations (i.e. possible solutions) that are all consistent with observed data and prior information, e.g. (Mosegaard \& Tarantola, 1995). The posterior distribution of such non-Gaussian inverse problems may be highly multi-modal: i.e. realizations from the posterior probability distribution may be located at isolated clusters in the high dimensional space spanning all possible earth models. If such clusters exist, each cluster will correspond to a group of realizations with similar appearance, and each cluster may represent one geological scenario. For example, one cluster may represent a commercial viable reservoir while another cluster may represent a non-commercial viable reservoir. Using a probabilistic approach to inverse problems theory, the relative probability of each locale scenario can easily be quantified. This may be hugely beneficial in characterizing reservoir models of all kinds. It should be noted that this study does not intend to analyze the different geological scenarios, but demonstrates a technique to reveal their nature, which would be helpful in this respect.

In the following we will analyze the result of a probabilistic formulated inversion of a normal incidence seismic data set from the southern part of Jutland, Denmark. Initially we will demonstrate that the posterior sample contains many quite different realizations. Then we use a clustering algorithm to locate multimodal clusters (i.e. similar posterior realizations). This analysis will be done both with respect to the realizations representing lithological units, and with respect to the realization's corresponding acoustic impedance parameterization. Finally the analysis obtained from the two different ways of parameterizing data will be compared.

\section{The inverse problem}

Figure 1 shows 26000 realizations resulting from sampling of the posterior distribution for a probabilistically formulated inversion of a reflection seismic data set from of the Zechstein unit in the southern part of Jutland, Denmark (Cordua, Gulbrandsen, Hansen, \& Mosegaard, submitted). This distribution is based on a prior distribution that is statistically consistent with geological scenes observed in borehole logs. Hence, the posterior realizations are all consistent with both the 
observed geological scenes and the observed seismic data (within the expected data uncertainty). A presentation of the inversion method used to obtain the posterior realizations is outside the scope of this paper. However, it can be assumed that the method in fact samples the true posterior distribution. Later we actually show that the method in fact revisits the same modes of the posterior distributions several times during the sampling period.

In Figure 1 it can be seen that this highly underdetermined seismic inverse problem has several possible solutions of different nature. Figure 1 further suggests some clusters of similar posterior realizations. In this study, we try to quantify this apparent clustering to get a better understanding of how the realizations actually are distributed. This is done using the unsupervised clustering method called K-means. In addition to provide a better visual representation of the results, the clustering algorithm allow quantifying the probability of the possible geological scenarios.

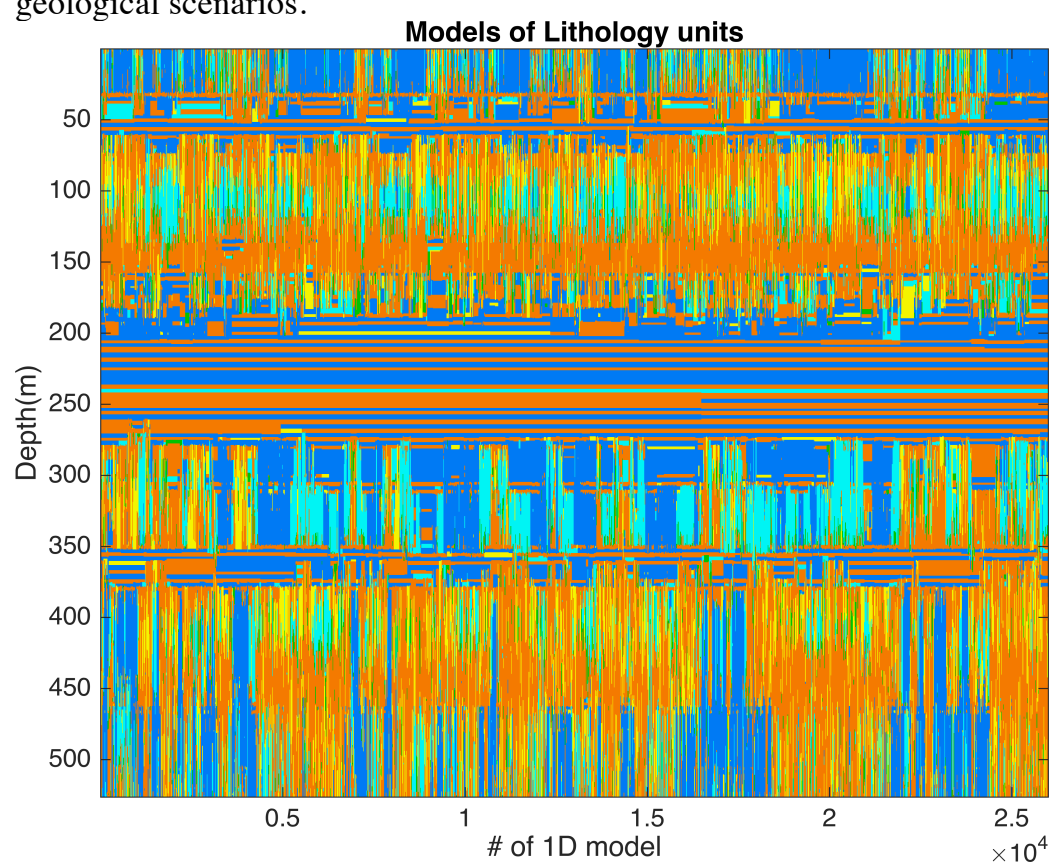

Figure 1 The figure shows 26000 1D lithology profiles resulting from an inversion of reflection seismic data. The colors blue, turquoise, green, yellow and orange represents the lithology units Halite, Limestone, Lamination, 50/50 Anhydrite and limestone, and Anhydrite respectively.

Figure 1 represents one way of displaying the posterior realizations. Each color represents a lithological unit. Another way could be to parameterize these units with their elastic property, acoustic impedance (AI). Table 1 shows the relation between the lithological units and their corresponding AI-values, as used in the inversion algorithm (Cordua, Gulbrandsen, Hansen, \& Mosegaard, submitted). 
Table 1: The different lithological units and the corresponding AI-values as used in (Cordua, Gulbrandsen, Hansen, \& Mosegaard, submitted)

\begin{tabular}{ll}
\hline Lithological units (color Figure 1) & AI-values $\left[\mathbf{k g} / \mathbf{m}^{\mathbf{2}} \mathbf{s}\right]$ \\
\hline Halite (blue) & $1.0^{*} 10^{7}$ \\
\hline Dolomitic Limestone (turquoise) & $1.2^{*} 10^{7}$ \\
\hline Lamination (green) & $1.4^{*} 10^{7}$ \\
\hline $\mathbf{5 0 / 5 0 L i m e s t o n e / A n h y d r i t e ~ ( y e l l o w ) ~}$ & $1.6^{*} 10^{7}$ \\
\hline Anhydrite (orange) & $1.8^{*} 10^{7}$ \\
\hline
\end{tabular}

\section{Locating cluster specific geological scenarios}

The task is now to group all the individual posterior realizations into different clusters. A general description of a cluster is difficult, and no unique definition exists. However, it can be valuable to think of clusters as groups of which all members are closer together with the other members from the same group than members from other groups, even though this not always has to be a mathematical fact. There are several ways of defining these mutual distances and several different clustering algorithms exist. There is no correct algorithm, since the different methods represents different ways of analyzing the data, and the different methods should be decided based on the problem. In this study, the K-means clustering algorithm will be used to group the posterior realizations both with respect to the lithological units, and with respect to the acoustic impedance values.

\section{K-means Clustering}

Assuming the number of clusters, $K$, is given, the aim is to group the $D$ dimensional data $\mathbf{x}=\left\{\mathbf{x}_{1}, \mathbf{x}_{2}, \ldots \mathbf{x}_{\mathbf{N}}\right\}$ into the $K$ clusters. The clusters are represented by the $D$-dimensional vectors $\boldsymbol{\mu}_{\mathrm{k}}$, where $k=1 \ldots K$, and each vector $\boldsymbol{\mu}_{\mathbf{k}}$ is a prototype vector for the $\mathrm{k}^{\text {th }}$ cluster (Bishop, 2006). More specifically, the prototype vectors represent the centers of each cluster, and hence the K-means clustering model is a centroid model. The objective goal of the method is to minimize the sum of the square distances between all points and their closest prototype vector. Mathematically this can be described as minimizing the objective function $J$ (Bishop, 2006):

$$
J=\sum_{n=1}^{N} \sum_{k=1}^{K} r_{n k}\left\|\boldsymbol{x}_{\boldsymbol{n}}-\boldsymbol{\mu}_{\boldsymbol{k}}\right\|^{2}
$$


where $r_{n k}$ is a set of binary indicator variables, $r_{n k} \in\{0,1\}$, each associated with one data point $\mathbf{x}_{\mathbf{n}}$, and stating which cluster each point is assigned to. In order to find the values for $r_{n k}$ and $\boldsymbol{\mu}_{\mathbf{k}}$ that minimizes equation (1) an iterative procedure is performed. Every iteration consists of two steps. The first step optimizes on the indicator variables and the next step on the cluster-centers. Prior to the simulation, $K$ prototype vectors are randomly chosen among the $D$-dimensional points $\mathbf{x}$ (note that there are several other ways to choose the starting points as well). In the first step of the iterations, each point is assigned a cluster based on the prototype vector, i.e. cluster center, they are closest to. This is in fact optimizing $J$ with respect to $r_{n k}$, since each data point is independent and taking the smallest distance between $\mathbf{x}_{\mathbf{n}}$ and $\boldsymbol{\mu}_{\mathbf{k}}$ will therefore minimize $J$. For the realizations of continuous variables, i.e. acoustic impedance the Euclidian distance is used and for categorical variables, i.e. the lithological units, the Hamming distance ${ }^{2}$ is used. The next step is to optimize on $\boldsymbol{\mu}_{\mathbf{k}}$ and since $J$ is a quadratic function of $\boldsymbol{\mu}_{\mathbf{k}}$ the optimal $\boldsymbol{\mu}_{\mathbf{k}}$ is found by setting the derivative of the objective function with respect to $\boldsymbol{\mu}_{\mathbf{k}}$ to zero:

$$
2 \sum_{n=1}^{N} r_{n k}\left(\boldsymbol{x}_{\boldsymbol{n}}-\boldsymbol{\mu}_{\boldsymbol{k}}\right)=0
$$

Solving equation (2) with respect to $\boldsymbol{\mu}_{\mathbf{k}}$ gives:

$$
\mu_{k}=\frac{\sum_{n} r_{n k} x_{n}}{r_{n k}}
$$

From equation (3) it is seen that $\boldsymbol{\mu}_{\mathbf{k}}$ represents the mean of all points within each cluster. The second step of each iteration is hence to compute the mean of all points assigned to each cluster, and define that new mean as the optimized cluster prototype. This procedure is repeated until the values converge. It should be noted, that depending on the data set, two different simulations (i.e. two different starting positions) might not converge to the same local minimum. You can never be sure if you reach the global minimum of the objective function, so it might be a good idea to run the simulations more then once.

\section{Gap-Statistic}

The K-means algorithm requires a predefined number of clusters. However, not knowing the complete nature of the dataset, this choice can be tricky. One way of approaching this issue could simply be to try different values, and visually examine the results. Another way could be to use a statistical approach to get an idea of which number of clusters that best represents the sample. In this study an

\footnotetext{
${ }^{2}$ The hamming distance is the number of position (coordinates/elements) that differs between two vectors of same length.
} 
analysis of the gap-statistic (Tibshirani, Walter, \& Hastie, 2001) is done. The idea of the gap-statistic is to find the number of clusters which standardize the comparison of $\log \left(\mathrm{W}_{\mathrm{k}}\right)$ with a distribution of no obvious clustering, when $\mathrm{W}_{\mathrm{k}}$ is defined as the within cluster dissimilarity:

$$
W_{k}=\sum_{r=1}^{K} \frac{1}{2 N_{r}} D_{r}
$$

where $N_{r}$ is the number of points within a clusters and $D_{r}$ is the distance between all points within cluster $r$. In this analysis the Euclidean distance is used for $D_{r}$ when the AI-data is analyzed, and the Hamming distance is used for the lithological units. The distribution with no obvious clustering is computed by taking the average of 20 simulations of uniformly distributed data within the range of the sample. Computing the within cluster dissimilarity of the uniformly distributed data, $U_{k}$, the Gap statistic is defined as:

$$
G=\log \left(U_{k}\right)-\log \left(W_{k}\right) .
$$

The optimal number of clusters is then the smallest number of $k$, which fulfill the criteria $G(k) \geq G(k+1)-s_{\{k+1\}}$, where $s_{\{k+1\}}$ is $\operatorname{std}\left(\log \left(U_{k}\right)\right) \sqrt{1+\left(\frac{1}{20}\right)}$ and 'std' denotes the standard deviation. Figure 2 shows the logarithm of the within cluster dissimilarity for both the sample (blue), and for the simulated data (red). This simulation is done with respect to the lithological units. As expected the blue curve decreases with an increasing number of clusters, while the red curve is more or less flat. In Figure 3 the Gap-curve is plotted. Based on this plot, it is seen that the optimal choice of clusters for this simulation is 15 (this is the first $\mathrm{k}$ where $G(k+1)-1$ std is less than $G(k))$.

This method is based on simulations, and the optimal number of clusters may therefore vary a little for different simulations. It should also be mentioned that the method is totally general and independent on any clustering method, so this analysis can be done with different clustering techniques. 


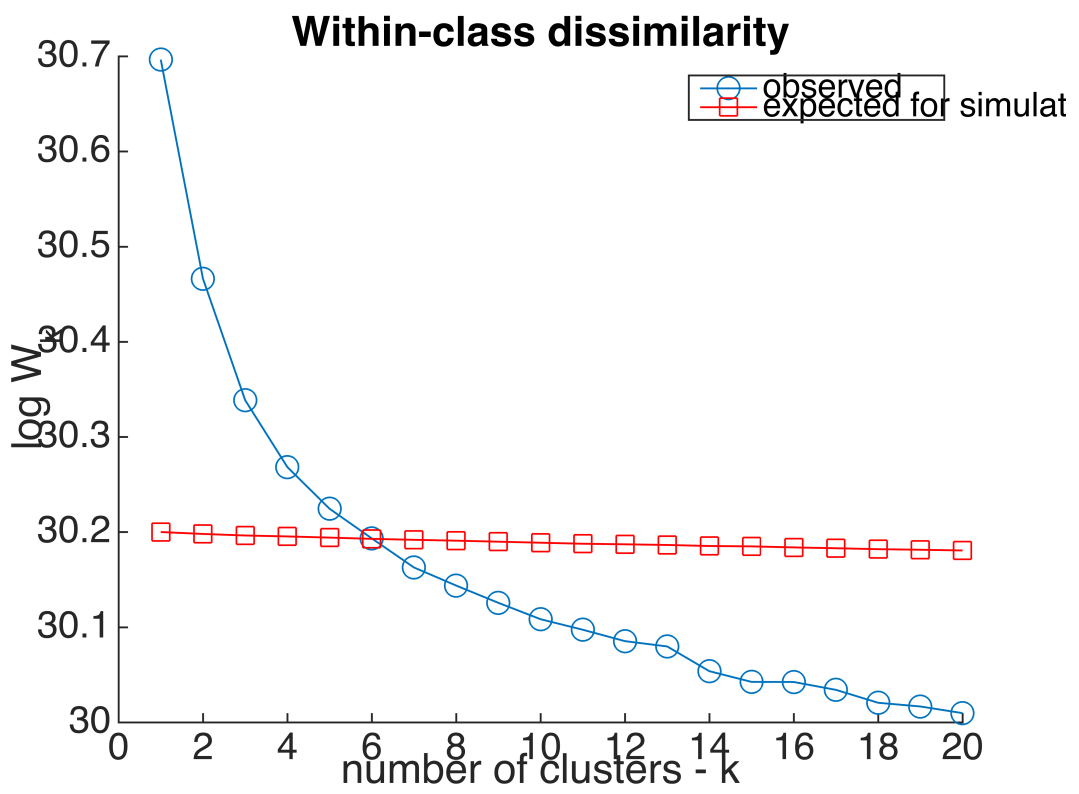

Figure 2 The figure shows the logarithmic within cluster dissimilarity of the observations (blue), and the simulated distribution (red) as functions of the number of clusters

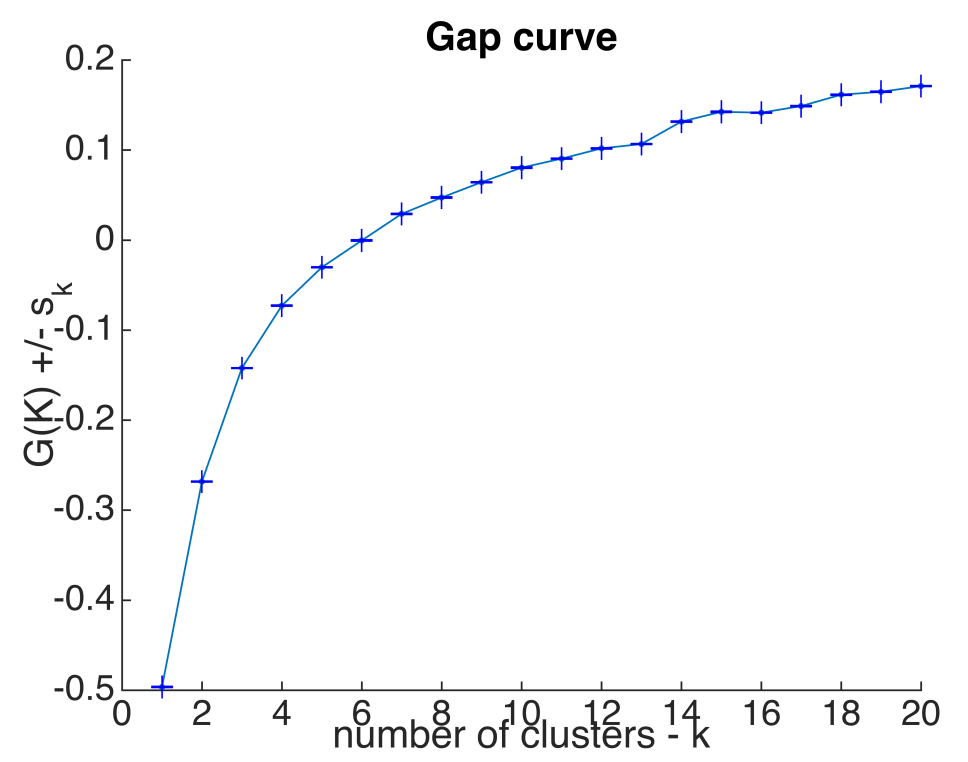

Figure 3 The figure shows the gap-statistics with its corresponding 1std-error-bars as a function of number of clusters. 


\section{Results}

Figure 2 and Figure 3 show the results from an analysis of the gap statistic of the posterior realizations in Figure 1 with respect to the lithological units. The gapstatistic simulations are however ambiguous, and the information from one simulation is hence not enough to base our choice of the optimal number of clusters. Figure 4 shows the histograms of 10 simulations of the gap-statistic using the K-means algorithm on both data sets. It is seen that the "optimal number of clusters" $\left(\mathrm{K}_{\mathrm{opt}}\right)$ varies between 6 and 20. It should be noted that the upper boundary of the simulation was set to 20 clusters, hence a result of $\mathrm{K}_{\mathrm{opt}}=20$ indicates that no optimal number of cluster was found within the test range (i.e., the optimal number of cluster may exist outside of this range). (Tibshirani, Walter, \& Hastie, 2001) states however that it can be important to examine the whole gapcurve, and not only the optimal choice. Figure 5 shows an example of a gap-curve representing a simulation where no optimal cluster numbers were found within the test range. It can however be seen that the criteria for concluding on an optimal number of clusters almost are met, both at $\mathrm{k}=10$ and $\mathrm{k}=16$.

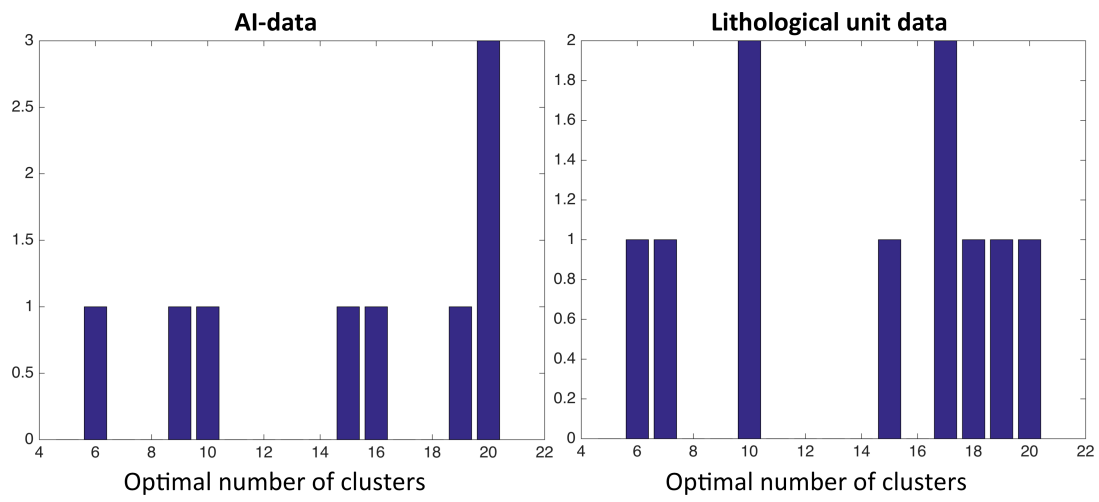

Figure 4: The figure shows the histograms of optimal number of clusters from 10 runs of the gap-statistic method using the K-means clustering algorithm on the AI-data (left plot) and the lithological unit data (right plot) respectively with respect to the optimum choice of $K$ (x-axis).

All the gap-curves for the 10 simulations for both data sets have been analyzed. This analysis combined with a visual examination of the clusters has resulted in the choice of running the K-means clustering algorithm with respect of grouping 15 clusters for both parameterizations. These results are presented in Figure 6 and Figure 7. The left panels of the two figures show which realizations from Figure 1 that is grouped in each cluster, and the right panels shows all these realizations put together to see the overall structure of each cluster. Note that the size of each plot in the right panel of the two figures not represent the actual size of the clusters. 
The relative sizes are printed above each plot, and represent the percentage of models belonging to those specific clusters.

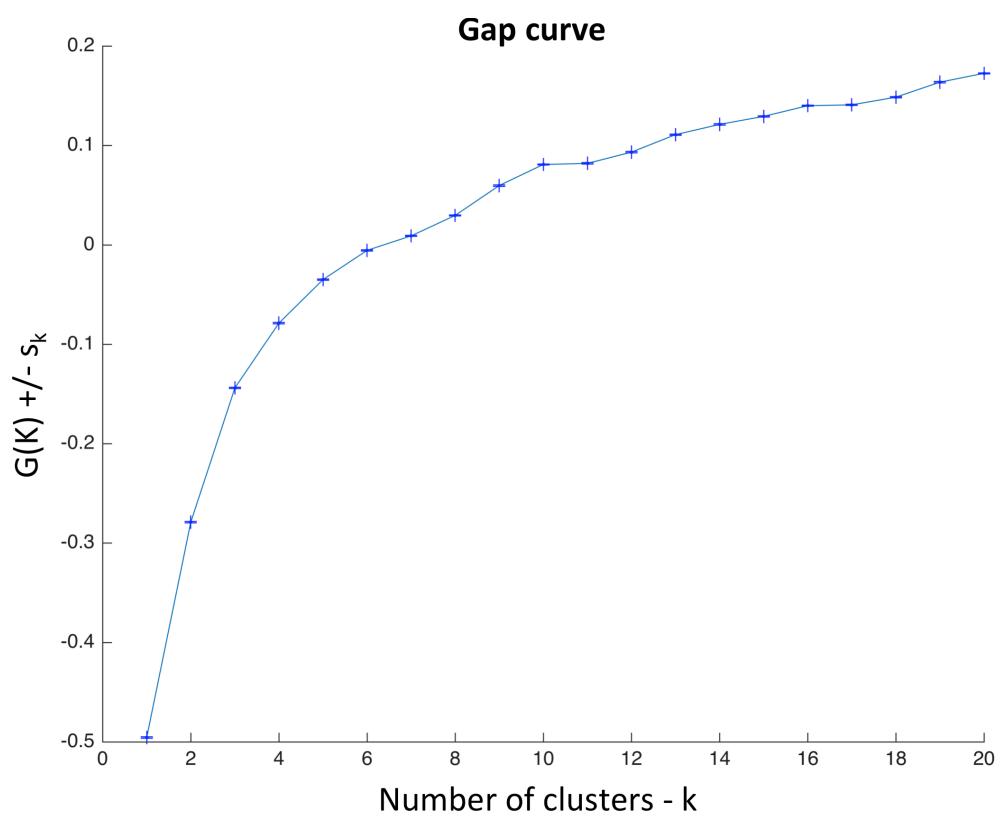

Figure 5: The gap curve from one simulation of the gap-statistic analysis using the Kmeans algorithm with respect to the AI-data.

Analyzing the plots in the left panels of Figure 6 and Figure 7, we can see that the realizations constituting the different clusters more or less are evenly distributed throughout the set of all realizations. Even though the inversion algorithm itself is outside the scope of this study, it can be mentioned as a curio that the distributions of models (seen in Figure 6 and Figure 7) from the same clusters indicate that the sampling algorithm actually visits the same modes of the posterior distributions several times during the sampling period. This is an underlying assumption of sampling the posterior distribution using the Metropolis algorithm. However, Figure 6 and Figure 7 demonstrate that this actually is the case. 

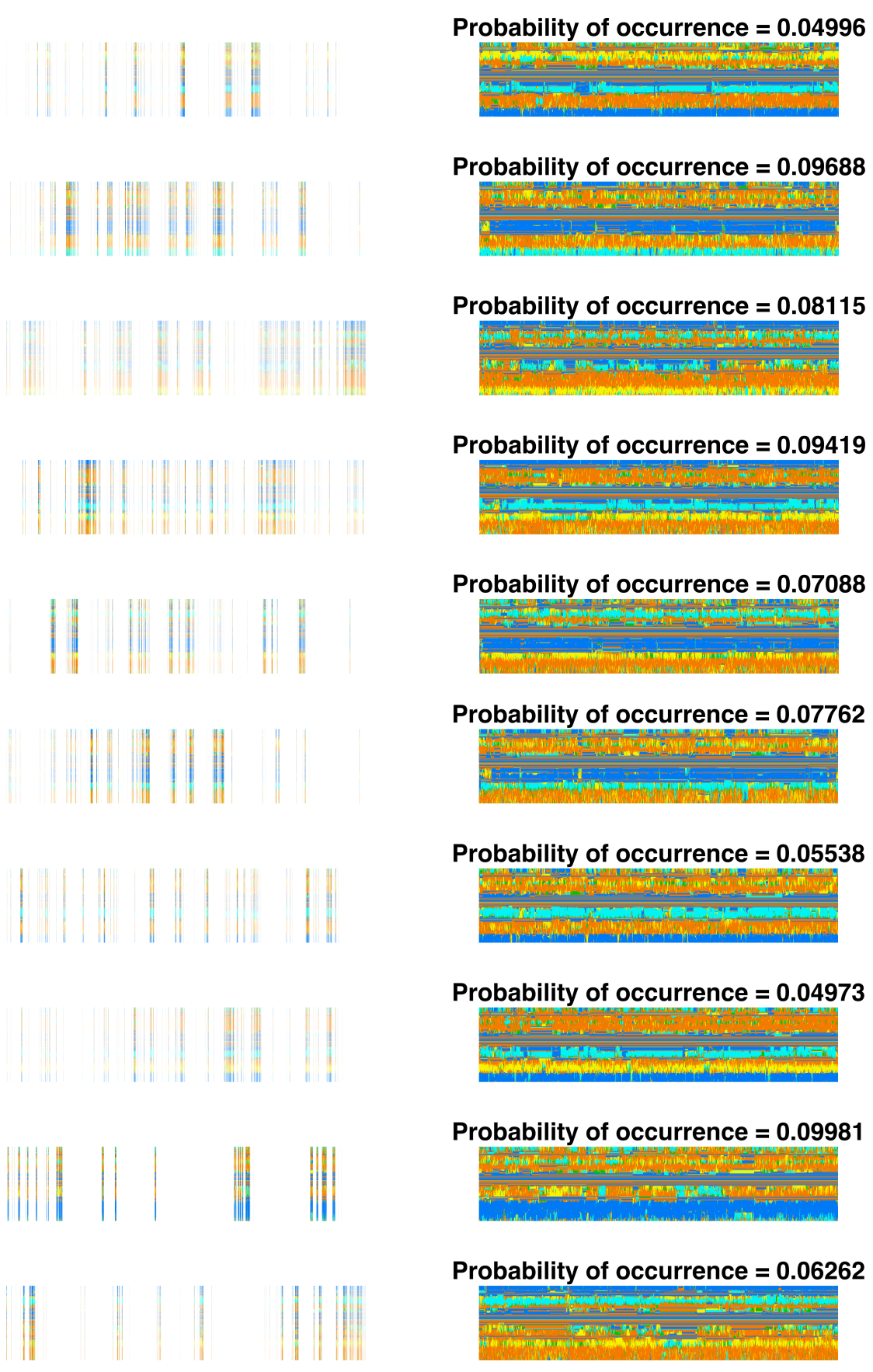

Probability of occurrence $\mathbf{=} \mathbf{0 . 0 8 1 1 5}$

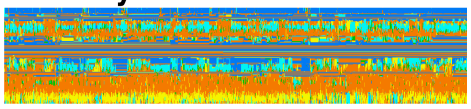

Probability of occurrence $\mathbf{=} \mathbf{0 . 0 9 4 1 9}$

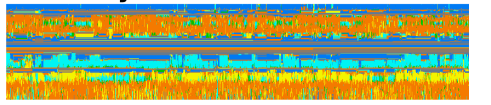

Probability of occurrence $\mathbf{= 0 . 0 7 0 8 8}$

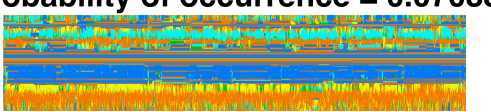

Probability of occurrence $\mathbf{=} \mathbf{0 . 0 7 7 6 2}$

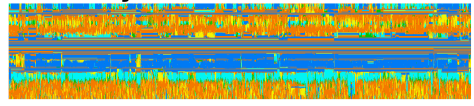

Probability of occurrence $\mathbf{=} \mathbf{0 . 0 5 5 3 8}$

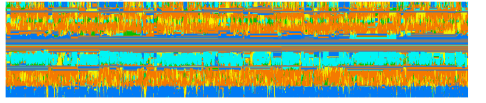

Probability of occurrence $\mathbf{= 0 . 0 4 9 7 3}$

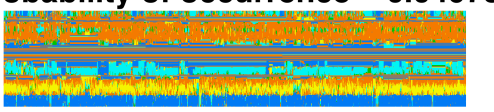

Probability of occurrence $\mathbf{= 0 . 0 9 9 8 1}$

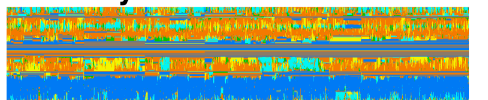

Probability of occurrence $\mathbf{=} \mathbf{0 . 0 6 2 6 2}$

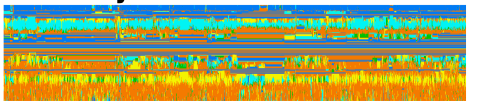



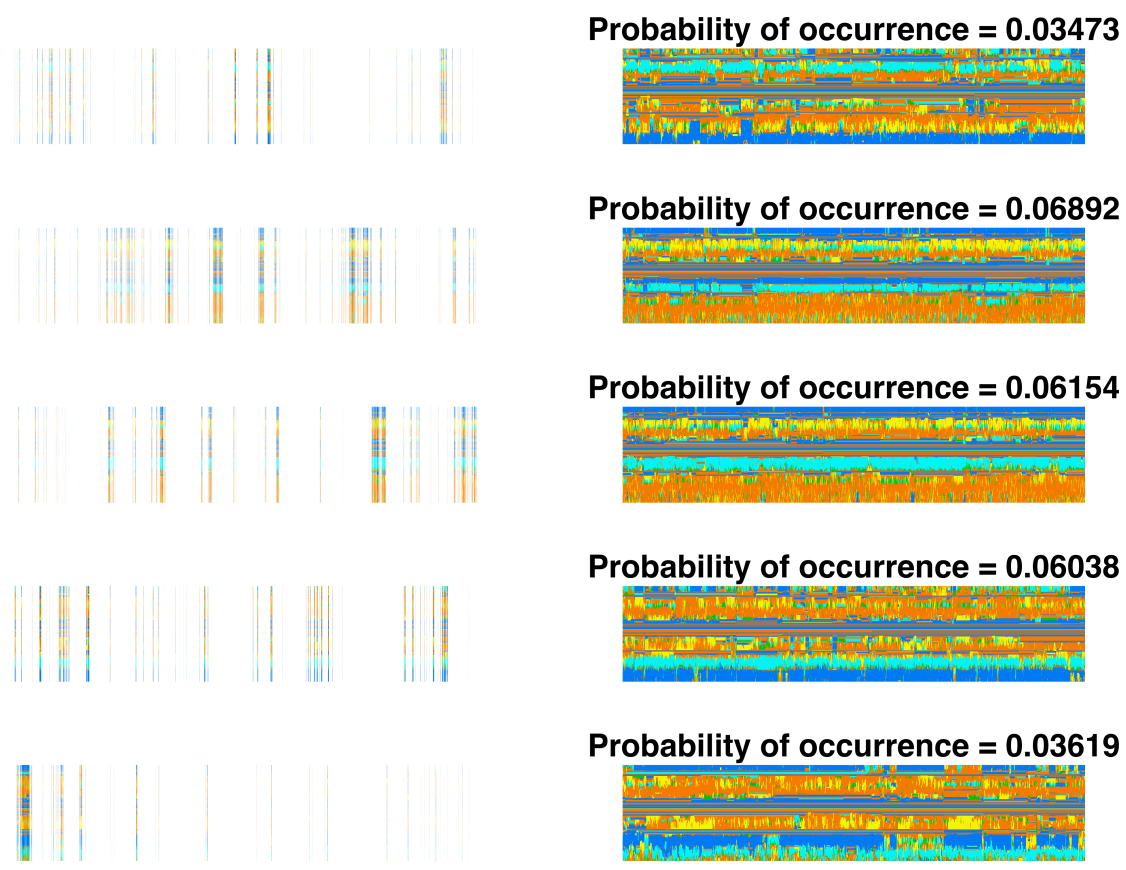

Probability of occurrence $\mathbf{= 0 . 0 6 8 9 2}$
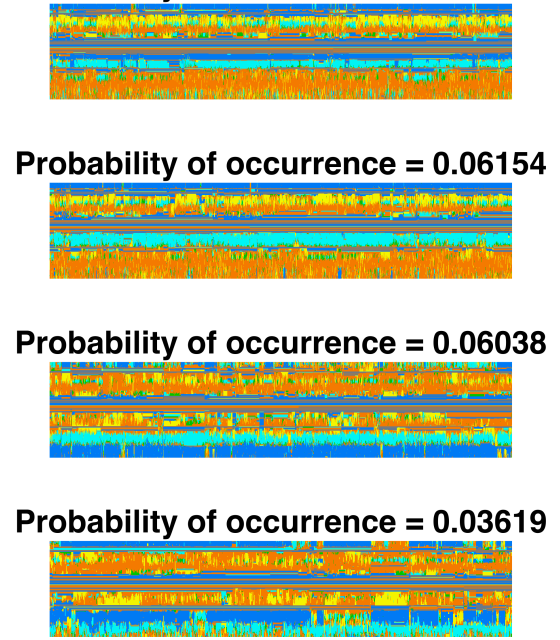

Figure 6 The figure shows the 15 clusters resulting from the K-means clustering of the lithology profiles in Figure 1. The left panel shows which models amongst the realizations that belong to each cluster and the right panel shows these models put together. The "Probability of occurrence" represents the number of models in each cluster relative to the whole sample. The lithology units are represented with the same colors as in Figure 1. 


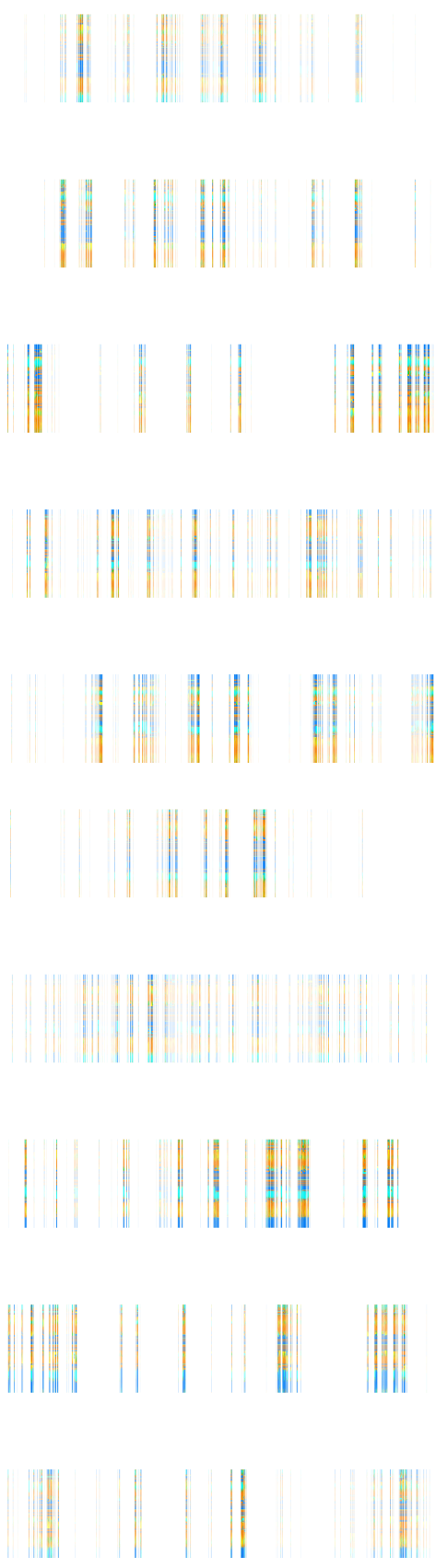

Probability of occurrence $\mathbf{=} \mathbf{0 . 0 5 4 5 0}$
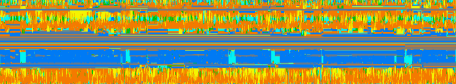

Probability of occurrence $\mathbf{= 0 . 0 6 5 5 8}$

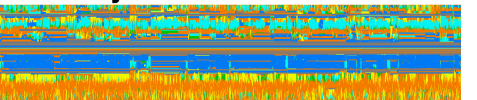

Probability of occurrence $\mathbf{= 0 . 0 8 3 8 8}$

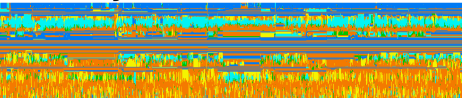

Probability of occurrence $=\mathbf{0 . 0 7 3 4 6}$

If $x$.

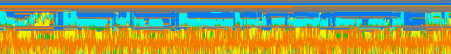

Probability of occurrence $\mathbf{=} \mathbf{0 . 0 8 7 7 3}$

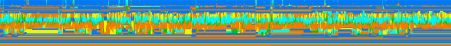

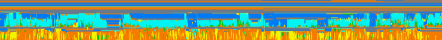

Probability of occurrence $\mathbf{=} \mathbf{0 . 0 4 1 8 8}$
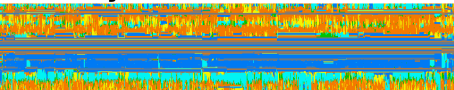

Probability of occurrence $=\mathbf{0 . 0 6 4 2 3}$

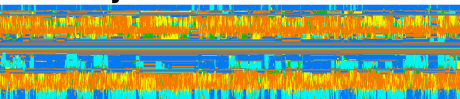

Probability of occurrence $\mathbf{=} \mathbf{0 . 1 0 1 1 9}$

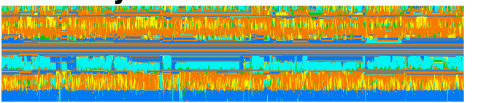

Probability of occurrence $\mathbf{= 0 . 0 8 7 7 7}$

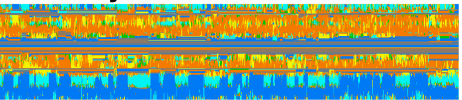

Probability of occurrence $\mathbf{=} \mathbf{0 . 0 5 5 2 7}$

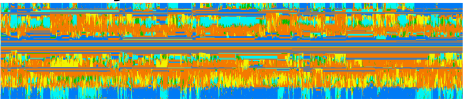



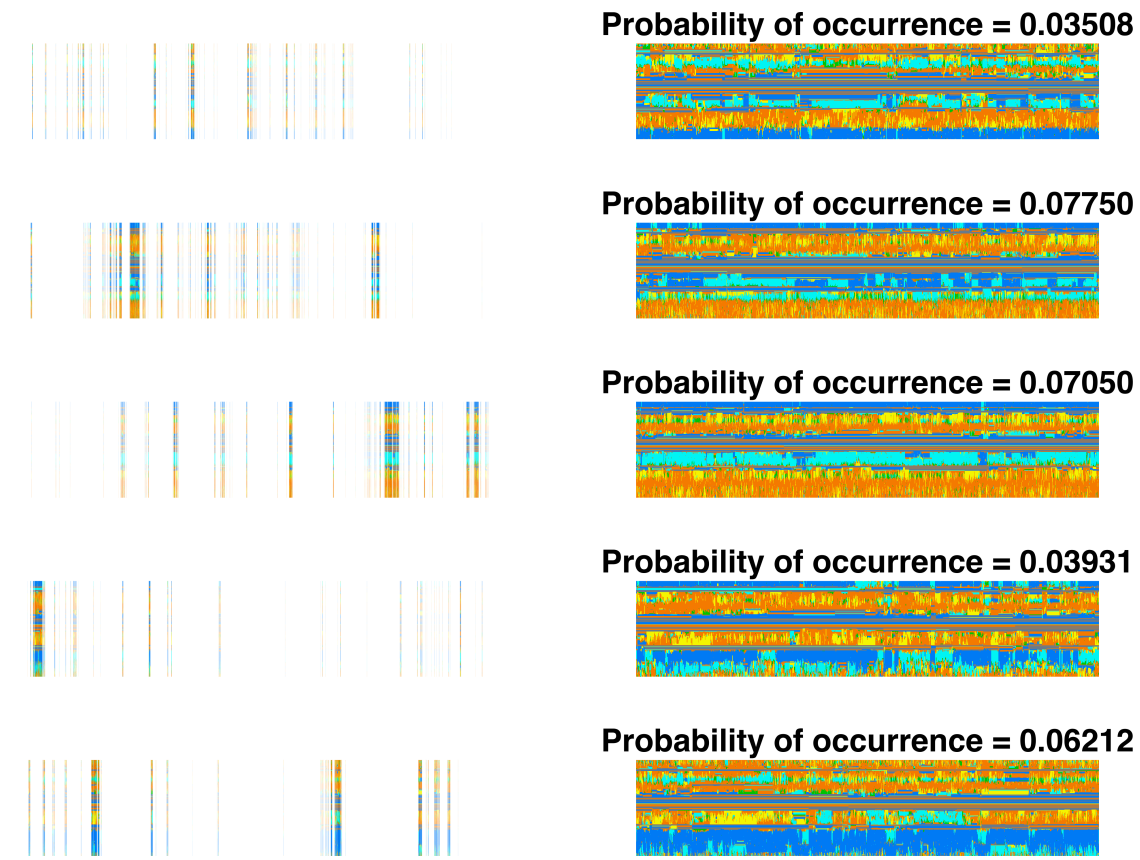

Probability of occurrence $\mathbf{=} \mathbf{0 . 0 7 7 5 0}$
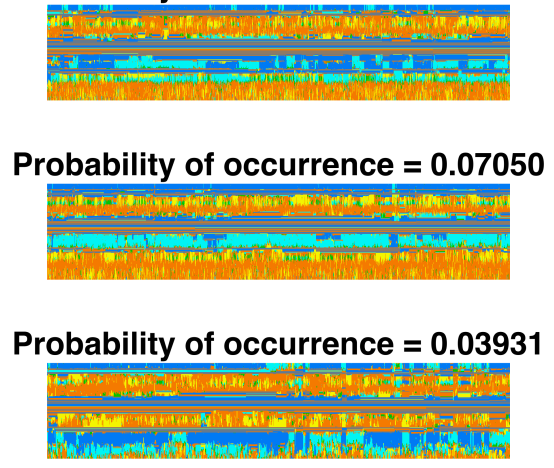

Probability of occurrence $\mathbf{=} \mathbf{0 . 0 6 2 1 2}$

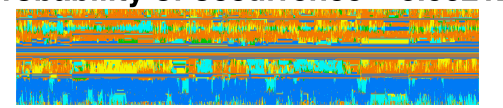

Figure 7: The figure shows the 15 clusters resulting from the K-means clustering of the AI realizations converted from the realizations shown in Figure 1, using the relation in Table 1. The left panel shows which models amongst the realizations that belong to each cluster and the right panel shows these models put together. The "Probability of occurrence" represents the number of models in each cluster relative to the whole sample. The lithology units are represented with the same colors as in Figure 1.

\section{Discussion}

\section{Other Clustering algorithms}

As stated earlier there is no such thing as a correct clustering algorithm. The different algorithms and methods can be thought of as looking at your data with different classes. In this specific study a few different agglomerative hierarchical clustering techniques also have been tried out. Hierarchical clustering does not, unlike the K-means clustering, need a predefined number of clusters. Instead the hierarchical clustering techniques can be divided into two main groups, namely the agglomerative and divisive group (Everitt, Landau, \& Leese, 2001). The agglomerative techniques all consists of a series of fusion of the $N$ individual data points into groups, where the divisive techniques starts out with all points belonging to the same cluster, and successively separates all points into finer and 


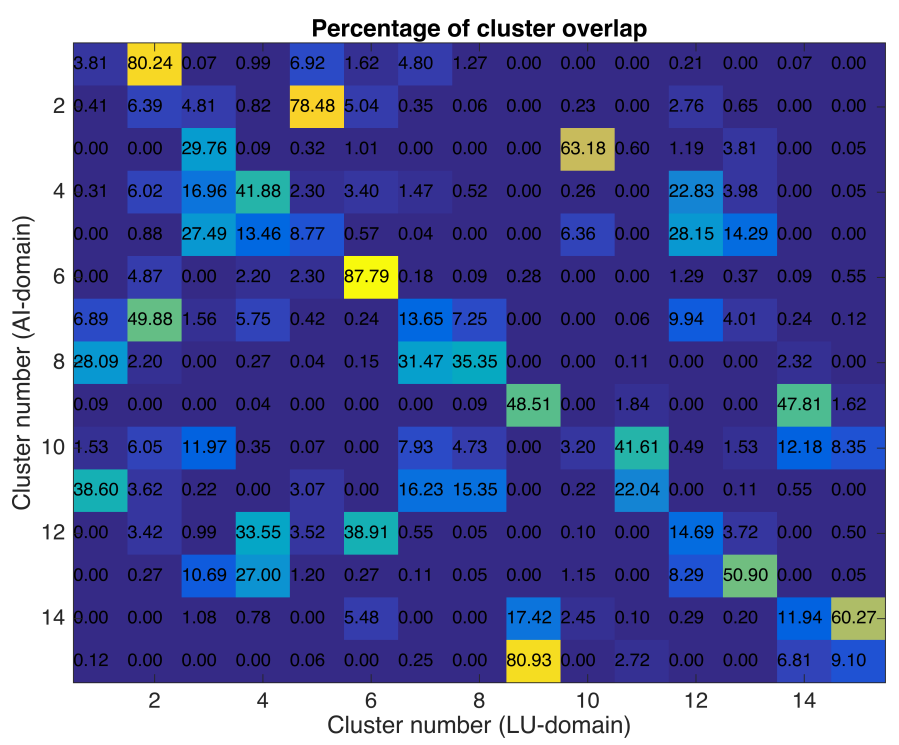

Figure 8 shows the percentage of models overlapping between the 15 clusters of the AI realizations (y-axis) and the 15 clusters representing the lithological units (x-axis).

cluster 9 in Al-domain

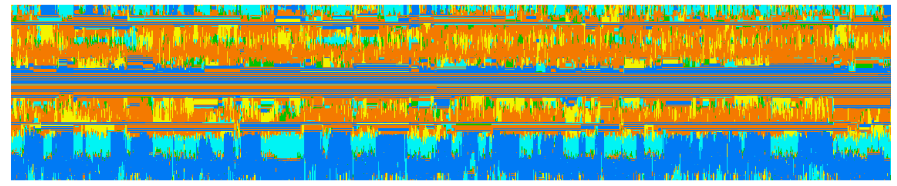

cluster 9 in LU-domain

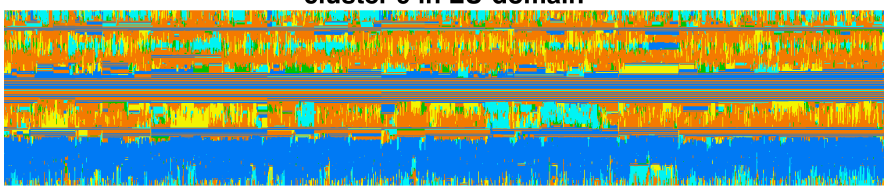

cluster 14 in LU-domain

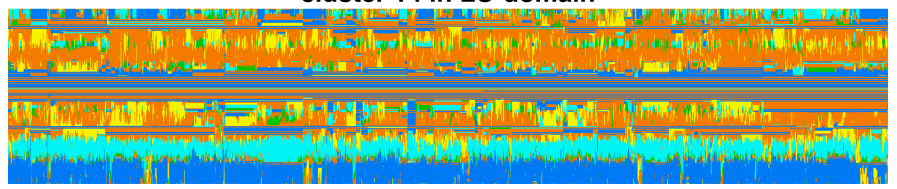

Figure 9: The upper plot shows the models representing cluster 9 in the AI-domain. The middle and lower plot shows cluster number 9 and 14 from the LU-domain.

finer groups. The merging or splitting of clusters is done with respect to different similarity/dissimilarity or distance measures between different clusters. All the different ways of measuring distances give rise to the different clustering methods. For the dataset in this study only techniques representing the agglomerative group 
have been tested, namely the single linkage, complete linkage, average linkage, and centroid linkage. None of the results perform as well as the K-means method for this study, which is a statement purely based on visual inspection of the clusters. None of these results will be presented here since the comparison of the different methods is outside the scope of this paper. Distribution models such as e.g. the Expectation Maximization (assuming data can be described as a Gaussian mixture model) are not considered in this study since they are unsuited for clustering categorical variables.

\section{Comparison of clustering results}

The importance of being able to cluster the data in the lithological unit (LU) domain is illustrated in Figure 8. The figure displays the percentage overlap between realizations belonging to the different clusters arising from applying the K-means clustering algorithm on the AI-data (vertical axis) and the lithological units (horizontal axis), respectively. As an example, it is seen from Figure 8 that cluster 15 in the AI-domain is pretty well represented in the lithological unit LUdomain. $80.9 \%$ of all realizations in cluster 6 from the AI-domain are grouped together in one cluster, namely number 9 , in the LU-domain. The rest of the realizations are mainly distributed between cluster 14 and 15 , with $6,8 \%$ and $9,1 \%$ respectively. If we, however, look at cluster 9 in the AI-domain, we can see that the models belonging to this cluster is split between two different clusters in the LU-domain, namely cluster number 9 and 14 with 48,5\% and 47,8\% respectively. These clusters are plotted in Figure 9. From the deeper part of the realizations displayed in Figure 9 it is clear that the clustering algorithm distinguish between the blue (halite) and the turquoise (Limestone) when applied to the LU-domain, but cluster realizations with both these sections together in the AI-domain. This is because these models are much closer together in the continuous parameter space, than in the discrete parameter space, where the distance is the same between all the categorical variables. It should be noted that the clustering simulations for the two data sets have the same starting points, i.e. the simulations starts in the same random realizations. This is important to emphasize, since it suggests that the differences illustrated in Figure 8 are actual differences of the two domains and not differences due to two different local minima of the objective function (eq. (1)).

\section{Conclusion}

We have shown that K-means clustering can be used to get a better understanding of how the multimodal landscape representing the solution space of a 1D reflection seismic inversion is distributed. By clustering the posterior realizations we get a much clearer picture of the potential geological scenarios and the 
probabilistic distribution between them. The combination of probabilistic inverse problems and cluster analysis thus allow us to perform scenario-based inversion of reflection seismic data.

It is however important to emphasize that the posterior probability distributions changes for the different parameterizations, which in turn can result in very different multimodal landscapes. Clustering the same realizations with different parameterization will hence result in different clusters, and it is important to know what is analyzed. In this study we show that one cluster in the AI-domain actually is represented in two different geological scenarios, and it is hence important to analyze the domain representing the parameterization of interest.

\section{Bibliography}

Bishop, C. M. (2006). Pattern Recognition and Machine Learning . Springer.

Bosch, M., Rodrigues, J., Navarro, A., \& Díaz, M. (2007). A Monte Carlo approach to the joint estimation of reservoir and elastic parameters from seismic amplitudes. Geophysics , 72 (6), 029-039.

Buland, A., \& Omre, H. (2003). Bayesian linearized AVO inversion. Geophysics , 68 (1), 185-198.

Cordua, K. S., Gulbrandsen, M. L., Hansen, T. M., \& Mosegaard, K. (submitted). Constructing scene-consistent prior distributions using a Markov-Frequency-matching model: Applied to seismic inversion. GJI.

Everitt, B. S., Landau, S., \& Leese, M. (2001). Cluster Analysis. London: Arnold.

Hampson, D. P., Russell, B. H., \& Bankhead, B. (2005). Simultaneous inversion of prestack seismic data. SEG Annual Meeting. Houston: Society of Exploration Geophysicists.

Jullum, M., \& Kolbjørnsen, O. (2016). A Gaussian-based framework for local Bayesian inversion of geophysical data to rock properties. GEOPHYSICS , 81 (3), R75-R87.

Larsen, A. L., Ulvmoen, M., Omre, H., \& Buland, A. (2006). Bayesian lithology/fluid prediction and simulation on the basis of a Markov-chain prior model. GEOPHYSICS, R69-R78.

Mosegaard, K., \& Tarantola, A. (1995). Monte Carlo sampling of solutions to inverse problems . Journal of Geophysical Research , 100 (B7), 12,431-12,447.

Tibshirani, R., Walter, G., \& Hastie, T. (2001). Estimating the number of clusters in a data set via the gap statistic. The Journal of the Royal Statistical Society , 63, part 2, 411423.

Ulvemoen, M., \& Omre, H. (2010). Improved resolution in Bayesian lithology/fluid inversion from prestack seismic data and well observations: Part 1-Methodology. Geophysics , 75 (2), R21-R35.

Zunino, A., Mosegaard, K., Lange, K., Melnikova, Y., \& Hansen, T. M. (2014). Monte Carlo reservoir analysis combining seismic reflection data and informed priors. Geophysics , R31-R41. 